\title{
Considerations in the management of congenital cranial dermoid cysts
}

\author{
Syed Khalid, BS, ${ }^{1,2}$ and John Ruge, MD1 \\ 1Division of Pediatric Neurosurgery, Advocate Children's Hospital, Park Ridge; and ${ }^{2}$ Chicago Medical School, North Chicago, \\ Illinois
}

\begin{abstract}
OBJECTIVE Congenital dermoid cysts (CDCs) develop from the entrapment of the surface ectoderm along the lines of embryonic fusion and have a capacity to grow. Given this capacity for continual expansion, the timing of removal and anticipation of possible epidural extension is important.
\end{abstract}

METHODS The authors retrospectively reviewed records of patients with the diagnosis of dermoid cyst presenting over a period of 10 years. Baseline characteristics, histological reports, and surgical records were collected and analyzed. Only those patients with histological confirmation of dermoid cyst were included in the study.

RESULTS One hundred fifty-nine (64 male and 95 female) patients were studied. The average age at the time of surgery ranged from 1 month to 63 years (mean $3.55 \pm 9.58$ years, median 11 months). Eighteen (11.3\%) CDCs were in the midline anterior fontanelle, $52(32.7 \%)$ were frontozygomatic, 14 (8.8\%) occurred along the coronal sutures, and $75(47.2 \%)$ occurred along the lambdoid sutures. In $74(46.5 \%)$ cases the cysts were found to insignificantly erode the cranium, $30(18.9 \%)$ eroded through a partial thickness of the cranium, $47(29.6 \%)$ eroded through the full thickness of the cranium, and $8(5.0 \%)$ eroded through the full thickness of the cranium and demonstrated epidural extension. The study population showed female predominance $(n=95,59.7 \%)$. Although cyst location and patient sex were not found to vary significantly with cranial involvement $(p=0.196$ and $p=0.066$, respectively), delay in time to surgery did vary significantly $(p<0.00001)$.

CONCLUSIONS Congenital cranial dermoid cysts found in infants and children are best removed early.

https://thejns.org/doi/abs/10.3171/2017.2.PEDS16701

KEY WORDS congenital dermoid cysts; epidural extension; cranial erosion; oncology

$\mathrm{C}$ ONGENITAL dermoid cysts (CDCs) develop from the entrapment of the surface ectoderm along the lines of embryonic fusion. ${ }^{9,10,12}$ Similarly, CDCs, on histological analysis, exhibit a well-developed fibrous wall lined by stratified squamous epithelium and contain a core of mesoderm-derived tissues such as glands and follicles. ${ }^{3,49}$ These lesions occur frequently on the head and may be recognized at birth or present later in life given their persistent nature and capacity to grow., ${ }^{1,7}$ These masses characteristically enlarge very slowly and ultimately become symptomatic as a result of enlargement, rupture, and even, in some cases, extension into surrounding structures. ${ }^{3,8}$

Given their capacity for growth, issues arise over how best to manage a solitary lump on a child's head when it is thought to be a CDC. One hundred fifty-nine cases of CDCs were analyzed, reporting the location of occurrence and the extent of cranial and epidural involvement.

\section{Methods}

After approval was obtained from the institutional review board, the electronic medical records were used to identify all patients in whom a dermoid cyst of the head was diagnosed between January 2005 and December 2015. Their medical histories were reviewed for demographic information, age at the time of surgery, diagnostic techniques, clinical features, treatment, and outcomes. All reported mass locations were confirmed intraoperatively and classified appropriately, irrespective of laterality, if applicable. Tissue specimens were available for all reported patients, and the histological features of the tumor were reviewed and confirmed by a pathologist. Any cases that could not be histologically confirmed were excluded. Each patient's primary pediatrician was asked to note any changes at 1 year, so as to recommend the return of the patient if appropriate. 


\section{Definition of Variables, Treatment, and Outcomes}

Demographic, clinical, and radiographic data were retrieved from electronic patient records. Time to surgery was defined by patient age at the time of surgery. The CDCs were defined on the basis of their location: midline anterior fontanelle, frontozygomatic, coronal sutures, and lambdoid sutures. Cranial erosion was defined as insignificant if there was no observable erosion of the cranium; partial-thickness erosion was defined as an erosion into the cranium through which the dura mater could not be observed. Likewise, full-thickness erosion was defined as cases in which the dura could be observed through the erosion, and full-thickness with epidural extension was used in the cases in which the CDC was found to be depressing and causing thinning of the dura (Fig. 1).

\section{Data Analysis}

The Student t-test was used for continuous variables, and the chi-square test was used for categorical variables. All analyses were performed to reflect the entire cohort. Statistical significance was defined as $\mathrm{p}<0.05$, and all statistical analyses were performed using R Statistical Software (version 3.1.1, 2013).

\section{Results}

One hundred fifty-nine (64 male and 95 female) patients were studied. The average age at the time of surgery ranged from 1 month to 63 years (mean $3.55 \pm 9.58$ years, median 11 months). The distribution of the cysts is shown in Table 1; $18(11.3 \%)$ CDCs were in the midline anterior fontanelle, $52(32.7 \%)$ were frontozygomatic, 14 (8.8\%) occurred along the coronal sutures, and $75(47.2 \%)$ occurred along the lambdoid sutures. In $74(46.5 \%)$ cases the cysts were found to insignificantly erode through the cranium, $30(18.9 \%)$ eroded through a partial thickness of the cranium, $47(29.6 \%)$ eroded through the full thickness of the cranium, and 8 (5.0\%) eroded through the full thickness of the cranium and demonstrated epidural extension. Our population showed female predominance $(n=95,59.7 \%)$.

Of note, all patients found to have full-thickness cranial erosion with epidural extension were female, and all but one of their lesions was found in the lambdoid suture region. In those cases presenting with insignificant erosion of the cranium the lesions were excised via a direct approach. Those with more extensive cranial involvement were removed, taking care to resect all of the cyst capsule. Resection often involved curetting the bone margins and carefully resecting the cyst capsule from sometimes adherent dura. Significant defects in the bone were repaired with cranioplasty, using titanium mesh and self-setting calcium phosphate bone cement as deemed appropriate. There were no peri- or postoperative complications in any of the reported cases. The clinical diagnosis of all reported CDCs was confirmed histologically by pathological investigation. On follow-up, no recurrences were documented.

\section{Age at Surgery}

The time to surgery in our population is summarized in Table 1 and Fig. 2. Latency to surgery was found to vary

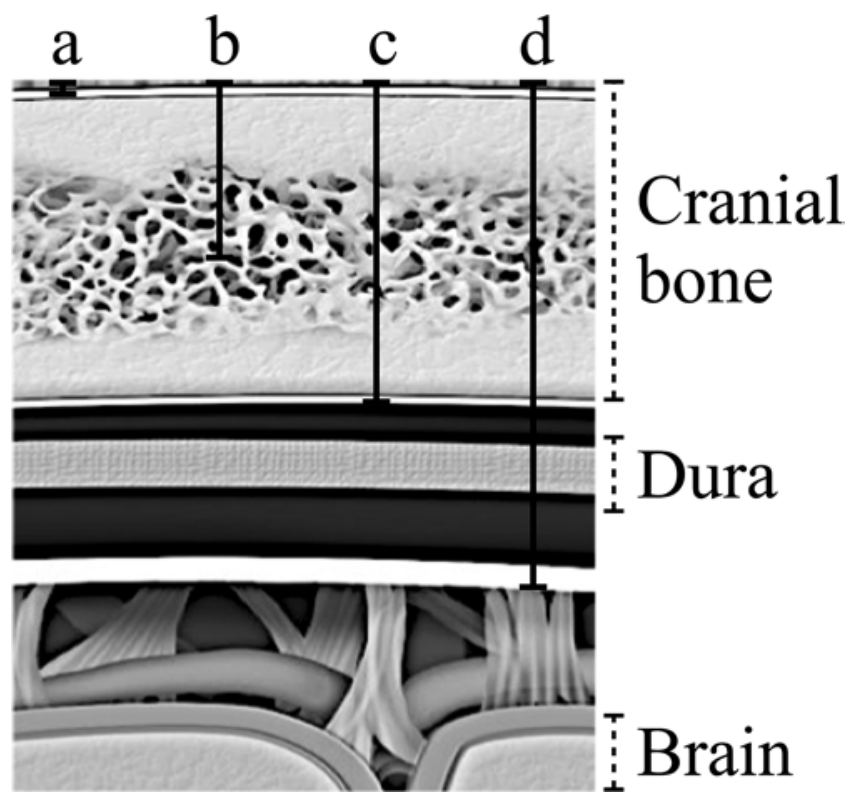

FIG. 1. Skull erosion definitions. Erosion was defined as insignificant (a), partial thickness (b), full thickness (c), and full thickness with epidural extension (d).

significantly with extent of cranial erosion and epidural extension $(\mathrm{p}<0.00001)$. In our population of 159 patients, $84(52.8 \%)$ underwent decisive treatment of their CDCs at $<1$ year of age (mean $0.57 \pm 0.22$ years); 61 (38.4\%) between 1 and 10 years (mean $2.08 \pm 1.46$ years); 3 (1.9\%) between 11 and 20 years (mean $15.33 \pm 3.79$ years); and $11(6.9 \%)$ at $>20$ years of age (mean $41.75 \pm 12.5$ years). Advancing age was clearly associated with extent of cranial erosion (statistically significant, $\mathrm{p}<0.00001$ ). The most extensive epidural extension and cranial deformity occurred in adults ( $\geq 18$ years). Five patients required cranioplasty and inpatient hospitalization.

Of the patients who underwent treatment at $<1$ year of age $(\mathrm{n}=84,52.8 \%), 42(50.0 \%)$ were found to have insignificant erosion of the cranium, $20(23.8 \%)$ had partialthickness erosion, $21(25.0 \%)$ had full-thickness erosion, and $1(1.2 \%)$ had full-thickness erosion that extended epidurally. In our population whose ages fell between 1 and 10 years of age $(n=61,38.4 \%), 26(42.6 \%)$ had insignificant cranial erosion, 8 (13.1\%) had partial-thickness erosion, 25 (40.9\%) had full-thickness erosion, and 2 (3.3\%) had full-thickness erosion with epidural extension. The group whose ages were between 11 and 20 years at the time of surgery $(\mathrm{n}=3,1.9 \%)$ and the population $>20$ years of age at the time of surgery $(n=11,6.9 \%)$ were found to have $0(0 \%)$ and $6(54.5 \%)$ patients, respectively, with insignificant cranial erosion; $2(66.7 \%)$ and $0(0.0 \%)$ with partial-thickness erosion; $0(0.0 \%)$ and $1(9.1 \%)$ with fullthickness cranial erosion; and 1 (33.3\%) and 4 (36.4\%) with full-thickness erosion with epidural extension.

\section{Cyst Locations}

The cyst locations and their corresponding extent of cranial erosion are summarized in Table 1 and Fig. 3. Specific 
TABLE 1. Descriptive characteristics of CDCs stratified by age at surgery, sex, location, and associated cranial involvement

\begin{tabular}{|c|c|c|c|c|c|c|}
\hline \multirow[b]{2}{*}{ Parameter } & \multirow[b]{2}{*}{ Total } & \multicolumn{4}{|c|}{ Cranial Erosion } & \multirow[b]{2}{*}{ p Value } \\
\hline & & Insignificant & Partial-Thickness & Full-Thickness & Full-Thickness w/ Epidural Extension & \\
\hline No. of patients & 159 & 74 & 30 & 47 & 8 & \\
\hline Age at surgery (\%) & & & & & & $<0.00001$ \\
\hline$<1 \mathrm{yr}$ & $84(52.8)$ & $42(56.8)$ & $20(66.7)$ & $21(44.7)$ & $1(12.5)$ & \\
\hline $1-10 \mathrm{yrs}$ & $61(38.4)$ & $26(35.1)$ & $8(26.7)$ & $25(53.2)$ & $2(25)$ & \\
\hline $11-20 \mathrm{yrs}$ & $3(1.9)$ & $0(0.0)$ & $2(6.7)$ & $0(0)$ & $1(12.5)$ & \\
\hline$>20 \mathrm{yrs}$ & $11(6.9)$ & $6(8.1)$ & $0(0.0)$ & $1(2.1)$ & $4(50.0)$ & \\
\hline $\operatorname{Sex}(\%)$ & & & & & & 0.066 \\
\hline Female & $95(59.7)$ & $47(63.5)$ & $17(56.7)$ & $23(48.9)$ & $8(100)$ & \\
\hline Male & $64(40.3)$ & $27(36.5)$ & $13(43.3)$ & $24(51.1)$ & $0(0.0)$ & \\
\hline Location of lesion (\%) & & & & & & 0.196 \\
\hline Midline anterior fontanelle & $18(11.3)$ & $8(10.8)$ & $6(20.0)$ & $3(6.4)$ & $1(12.5)$ & \\
\hline Frontozygomatic & $52(32.7)$ & 23 (31.1) & $8(26.7)$ & $20(42.6)$ & $1(12.5)$ & \\
\hline Coronal sutures & $14(8.8)$ & $5(6.8)$ & $2(6.7)$ & $7(14.9)$ & $0(0.0)$ & \\
\hline Lambdoid sutures & $75(47.2)$ & $38(51.4)$ & $14(46.7)$ & $17(36.2)$ & $6(75.0)$ & \\
\hline
\end{tabular}

cranial locations did not significantly vary with the extent of cranial erosion demonstrated by CDCs $(p=0.196)$. The most common location in our population was the lambdoid suture region (75 [47.2\%]). The majority of the CDCs found in this region (38 [50.7\%]) demonstrated an insignificant amount of cranial erosion, $14(18.7 \%)$ of these lesions demonstrated partial-thickness erosion, 17 (22.7\%) demonstrated full-thickness erosion, and $6(8.0 \%)$ demonstrated full-thickness erosion with epidural extension.

The second most common location of CDCs in our population was frontozygomatic $(\mathrm{n}=52,32.7 \%)$. The majority of these cases $(n=23,44.2 \%)$, eroded through the full thickness of the cranium, with 1 case $(1.9 \%)$ demonstrating full thickness of the cranial erosion with epidural extension. The rest of the cases were found to be in either the midline anterior fontanelle or coronal sutures $(n=18$ [11.3\%] and $n=14$ [8.8\%], respectively). Of these cases, the majority of the midline anterior fontanelle CDCs were found to demonstrate an insignificant amount of cranial erosion $(n=8,44.4 \%)$, whereas half of the coronal cases $(\mathrm{n}=7,50.0 \%$ ) were found to display full-thickness cranial erosion.

\section{Illustrative Case}

An 18-year-old, high-achieving young woman had had a painless lump over her right forehead since birth. The lump increased in size over the years, but caused her no symptoms. This patient suffered from frequent panic attacks and an MRI was obtained on presentation to our pediatric neurosurgical clinic (Fig. 4). Brain MRI demonstrated a $2.5-\mathrm{cm}$, well-circumscribed cranial mass causing mild pressure on her right frontal lobe (Fig. 4A). Additional imaging obtained using CT detailed the bony involvement (Fig. 4B).

This patient subsequently underwent craniectomy and removal of an intradiploic dermoid cyst with epidural extension. Cranioplasty was performed using titanium mesh and self-setting calcium phosphate bone cement (Fig. 4C and D). She made an uneventful recovery and is doing well.

\section{Discussion}

In our practice, we found 159 histologically verified cases of CDCs over a 10-year period. Eighty-five (53.4\%) of these cysts demonstrated some level of cranial involvement, and 55 cases $(34.6 \%)$ of CDCs were found to in-

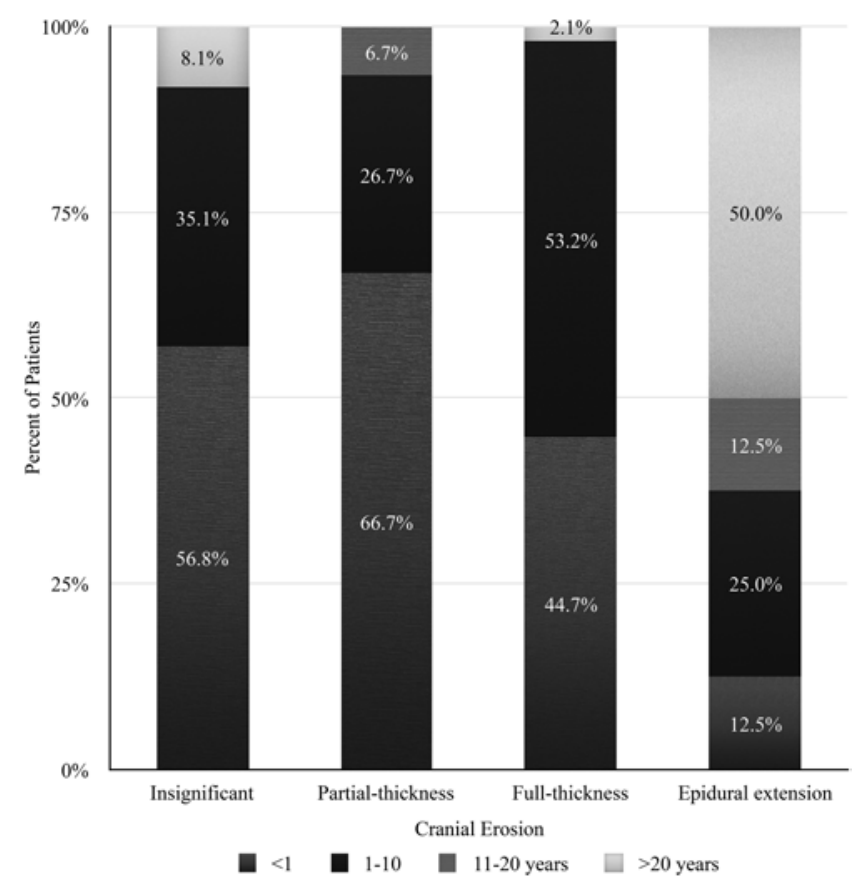

FIG. 2. Bar graph showing age at time of surgery and associated cranial involvement. 


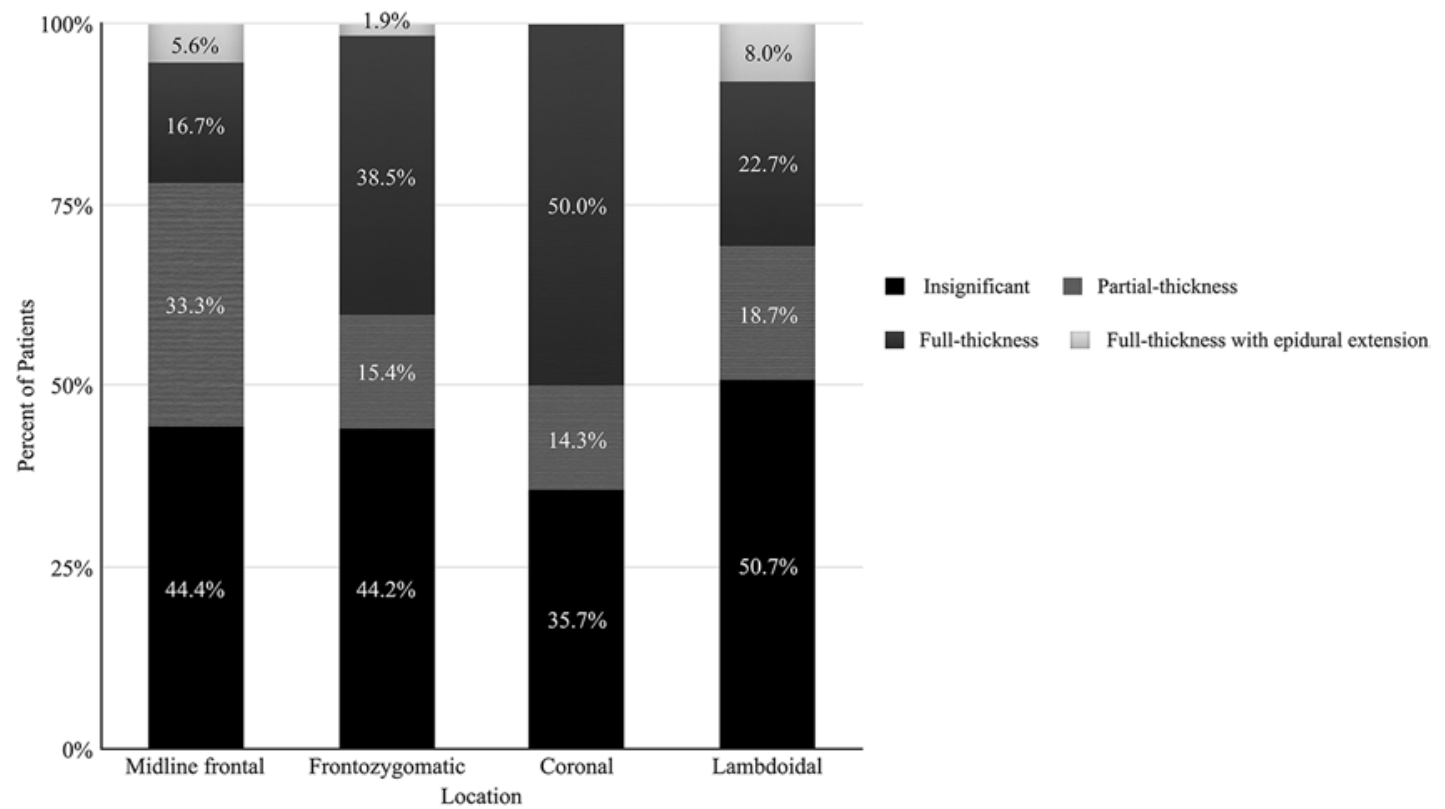

FIG. 3. Bar graph showing incidence of dermoid cyst location and associated cranial involvement.
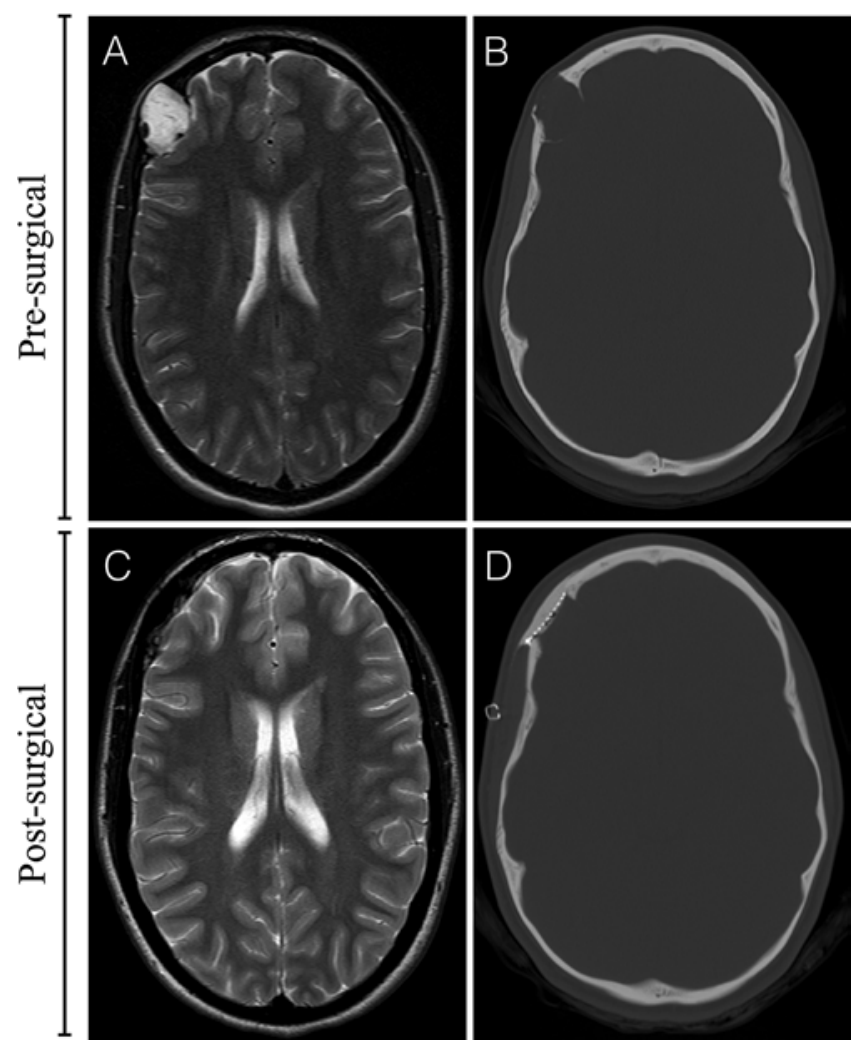

FIG. 4. Illustrative case. Presurgery and postsurgery imaging. Axial T2-weighted MRI study demonstrating a $2.5-\mathrm{cm}$, well-circumscribed cranial mass causing mild pressure on the patient's right frontal lobe $(\mathbf{A})$ and axial CT detailing the bony involvement (B) prior to resection. Axial T2-weighted MRI study (C) and axial CT (D) demonstrating successful resection of the cranial mass. volve the dura. Because CDCs possess the capacity for expansion, cranial erosion, and brain compression, we recommend resection. Early resection may avoid a more extensive operation. This is illustrated by the case presentation and our data, which illustrate that patients presenting later in life for treatment may be at an increased risk for full-thickness cranial erosion. The existence of completely penetrating lesions in the younger children suggests that more than latency is at work. We suspect that these cases represent instances in which the entrapment of the surface ectoderm along the lines of embryonic fusion has occurred anatomically deeper in origin as compared with their nonpenetrating counterparts.

As compared with the reported literature, our experience demonstrates that surgical complications after CDC removal are generally minor, and given the potential for adverse effects if left untreated, these patients should be treated in a timely manner. ${ }^{3,6,11,13}$

\section{Study Limitations}

We were unable to analyze volume or size of the CDC. Size may well influence the extent of cranial erosion and epidural extension. Our lack of volumetric analysis and the unknown baseline tumor size are limitations of our study. Our study was not prospective. Long-term followup was not possible beyond 1 year. Of note, our population does not represent the true incidence of the condition, but rather a measure of those presenting to our neurosurgical clinic.

\section{Conclusions}

In this study, cyst location and patient sex did not vary significantly with epidural extension $(\mathrm{p}=0.196$ and $\mathrm{p}=$ 0.066 , respectively). Age at the time of surgery did vary significantly with epidural extension of dermoid cysts $(\mathrm{p}<$ 
0.00001). The majority of the patients demonstrated some level of cranial erosion. Removal of all solitary masses is recommended, except for solitary lymph nodes. Patients who are young and who have solitary masses overlying cranial sutures undergo operation without preoperative imaging. In older patients or those with atypical location of the mass, MRI and/or CT studies are obtained prior to surgery. We believe that our zero-complication rate is in part due to management of these lesions by neurosurgeons trained to deal with dural adherence and epidural extension of mass lesions. Surgeons operating on dermoid cysts thus must be prepared to deal with the consequences of cranial defects and epidural extension, particularly when treating older patients. Likewise, early referral is recommended.

\section{References}

1. Anderson DJ, Dobson C, Berry RB: Nasal dermoid cysts in siblings. Int J Pediatr Otorhinolaryngol 48:155-159, 1998

2. Brownstein MH, Helwig EB: Subcutaneous dermoid cysts. Arch Dermatol 107:237-239, 1973

3. Charrier JB, Rouillon I, Roger G, Denoyelle F, Josset P, Garabedian EN: Craniofacial dermoids: an embryological theory unifying nasal dermoid sinus cysts. Cleft Palate Craniofac J 42:51-57, 2005

4. Crawford JK, Webster JP: Congenital dermoid cysts of the nose. Plast Reconstr Surg (1946) 9:235-260, 1952

5. de Aquino HB, de Miranda CC, de Britto Filho CA, Carelli $\mathrm{EF}$, Borges G: Congenital dermoid inclusion cyst over the anterior fontanel: report of three cases. Arq Neuropsiquiatr 61:448-452, 2003

6. Dutta S, Lorenz HP, Albanese CT: Endoscopic excision of benign forehead masses: a novel approach for pediatric general surgeons. J Pediatr Surg 41:1874-1878, 2006

7. McAvoy JM, Zuckerbraun L: Dermoid cysts of the head and neck in children. Arch Otolaryngol 102:529-531, 1976
8. Orozco-Covarrubias L, Lara-Carpio R, Saez-De-Ocariz M, Duran-McKinster C, Palacios-Lopez C, Ruiz-Maldonado R: Dermoid cysts: a report of 75 pediatric patients. Pediatr Dermatol 30:706-711, 2013

9. Pollard ZF, Harley RD, Calhoun J: Dermoid cysts in children. Pediatrics 57:379-382, 1976

10. Rubin G, Scienza R, Pasqualin A, Rosta L, Da Pian R: Craniocerebral epidermoids and dermoids. A review of 44 cases. Acta Neurochir (Wien) 97:1-16, 1989

11. Ruge JR, Tomita T, Naidich TP, McLone DG, Hahn YS: Scalp and calvarial masses in infants and children. Neurosurgery 22:1037-1042, 1988

12. Smirniotopoulos JG, Chiechi MV: Teratomas, dermoids, and epidermoids of the head and neck. Radiographics 15:14371455,1995

13. Weiss DD, Robson CD, Mulliken JB: Transnasal endoscopic excision of midline nasal dermoid from the anterior cranial base. Plast Reconstr Surg 102:2119-2123, 1998

\section{Disclosures}

The authors report no conflict of interest concerning the materials or methods used in this study or the findings specified in this paper.

\section{Author Contributions}

Conception and design: both authors. Acquisition of data: both authors. Analysis and interpretation of data: both authors. Drafting the article: both authors. Critically revising the article: both authors. Reviewed submitted version of manuscript: both authors. Approved the final version of the manuscript on behalf of both authors: Ruge. Statistical analysis: both authors. Study supervision: Ruge.

\section{Correspondence}

John Ruge, Department of Neurosurgery, Advocate Children's Hospital, 1675 Dempster St., Park Ridge, IL 60068. email: jruge22@gmail.com. 